\title{
Pooling Upper Respiratory Specimens for Rapid Mass Screening of COVID-19 by Real-Time RT-PCR
}

So Yeon Kim, ${ }^{1}$ Jaehyeon Lee, ${ }^{1}$ Heungsup Sung, Hyukmin Lee, Myung Guk Han, Cheon Kwon Yoo, Sang Won Lee, ${ }^{2}$ Ki Ho Hong ${ }^{2}$

To validate the specimen-pooling strategy for real-time reverse transcription PCR detection of severe acute respiratory syndrome coronavirus 2 , we generated different pools including positive specimens, reflecting the distribution of cycle threshold values at initial diagnosis. Cumulative sensitivities of tested pool sizes suggest pooling of $\leq 6$ specimens for surveillance by this method.

\footnotetext{
fter the first report of the coronavirus disease (COVID-19) outbreak in Wuhan, China (1), the World Health Organization announced pandemic status on March 11, 2020 (2). Real-time reverse transcription PCR (rRT-PCR) detection of the causative agent, severe acute respiratory syndrome coronavirus 2 (SARS-CoV-2), is a confirmatory diagnostic tool for COVID-19 (3).

A mass screening test for COVID-19 is urgently needed in South Korea because of the increasing number of confirmed cases in long-term care hospitals and public facilities, as well as imported cases. Testing specimens pooled before RNA extraction and subsequently retesting single specimens from positive pools is an efficient strategy for rapid mass screening as well as for increasing testing capacity and conserving resources.

Testing pooled specimens is a well-known method and has been used in blood banks worldwide to screen for infectious disease; however, only a few

Author affiliations: National Medical Center, Seoul, South Korea (S.Y. Kim); Jeonbuk National University Medical School and Hospital, Jeonju, South Korea (J. Lee); Asan Medical Center and University of Ulsan College of Medicine, Seoul (H. Sung); Yonsei University College of Medicine, Seoul (H. Lee); Korea Centers for Disease Control and Prevention, Cheongju (M.G. Han, S.W. Lee, C.K. Yoo); Seoul Medical Center, Seoul (K.H. Hong)

DOI: https://doi.org/10.3201/eid2610.201955
}

studies have evaluated specimen pooling for SARSCoV-2 (4,5; R. Hanel et al., unpub. data. https:/ / arxiv. org/abs/2003.09944v1; M.J. Farfan et al., unpub. data, https://doi.org/10.1101/2020.04.15.20067199). Therefore, we evaluated the pooling strategy for SARSCoV-2 testing using clinical specimens from 3 hospitals in South Korea: Seoul Medical Center and National Medical Center, both in Seoul, and Jeonbuk National University Hospital in Jeonju. The Institutional Review Boards of the hospitals approved this study. Written consent from participants was waived.

\section{The Study}

Pooled upper respiratory specimens were prepared from 50 individual SARS-CoV-2-positive specimens and 300 individual SARS-CoV-2-negative specimens. Either a single nasopharyngeal swab (NPS) or a nasopharyngeal and an oropharyngeal swab (NPS/OPS) were collected in an eNAT tube (Copan Italy, https:/ / www.copangroup.com). Laboratory diagnosis of SARS-CoV-2 infection was performed with all specimens using the following rRT-PCR kits targeting the $E$ and $R d R p$ genes: STANDARD $\mathrm{M} n \mathrm{nCoV}$ Real-time Detection (SD Biosensor, https://sdbiosensor.com) or PowerCheck 2019-nCoV Real-Time Detection (Kogene Biotech, https:// kogene.co.kr).

For the SARS-CoV-2-positive pooled specimens, we selected 50 individual SARS-CoV-2-positive specimens on the basis of the observed population distribution of cycle threshold $\left(C_{t}\right)$ values of rRT-PCR for patients confirmed positive during January 20-March 2, 2020 (Figure 1). We grouped the $C_{t}$ values into 8 strata, decided the sampling number adequate for each stratum, and selected a total of 50 specimens

\footnotetext{
${ }^{1}$ These first authors contributed equally to this article.

${ }^{2}$ These authors contributed equally to this article.
} 


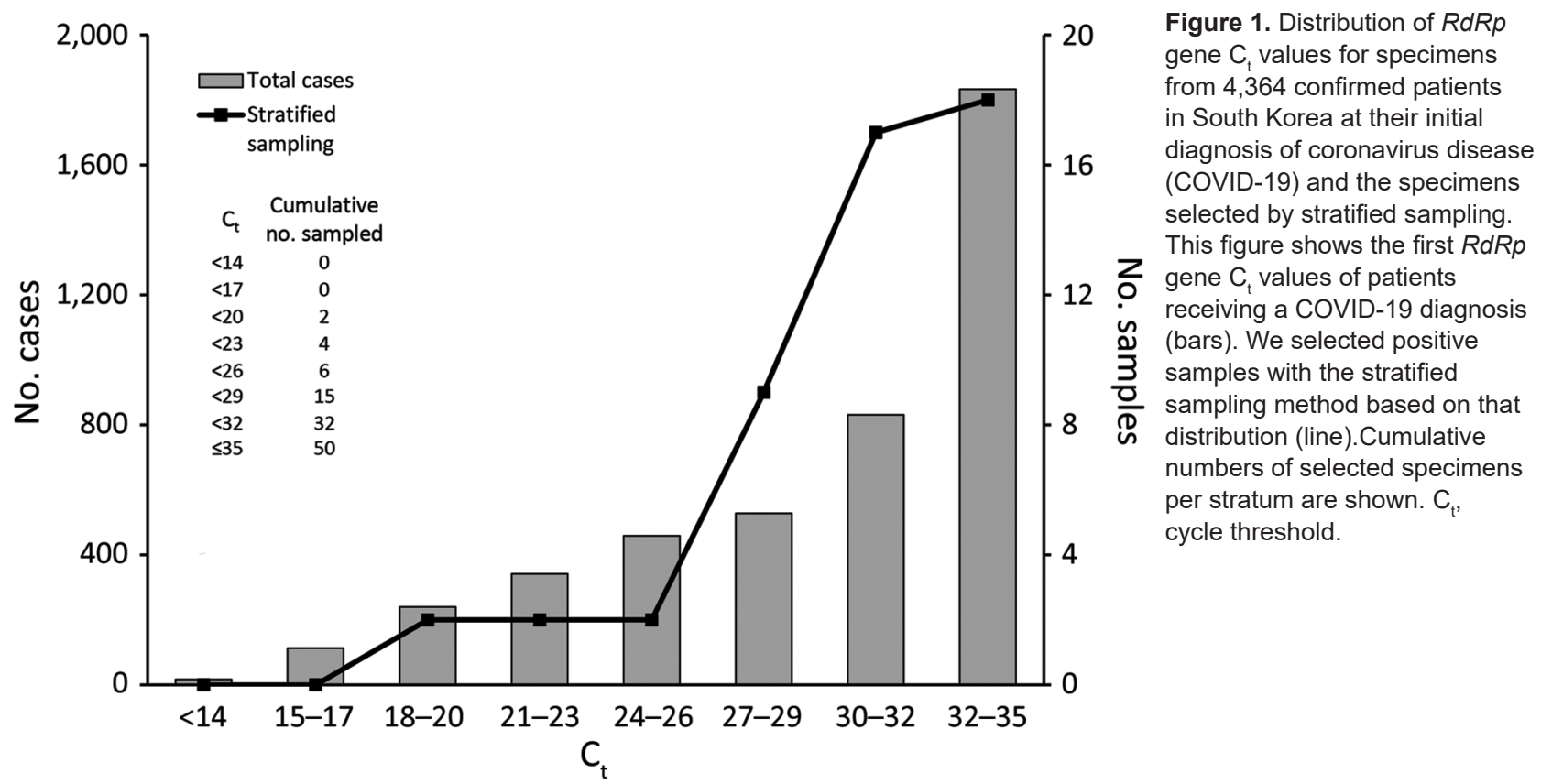

for 8 strata (Figure 1). We pooled the selected individual SARS-CoV-2-positive specimens with different numbers of SARS-CoV-2-negative specimens to generate 50 sets of pooled specimens in duplicate; the pool sizes of each set were $2,4,6,8,10$, and 16 . We prepared a total of 600 pooled specimens. To evaluate clinical specificity in SARS-CoV-2-negative pooled specimens, we randomly combined 16 specimens from 300 negative specimens and generated 60 negative pooled specimens (Appendix, https://wwwnc. cdc.gov/EID/article/26/10/20-1955-App1.pdf).

The following 3 automated RNA extraction systems were used: MagNa Pure 96 (Roche Diagnostics, https://www.roche.com), Real-prep (BioSewoom, www.biosewoom.com), and eMAG (bioMérieux, https://www.biomerieux.com). We followed the extraction protocol provided by each manufacturer with an input volume of $200 \mu \mathrm{L}$ and elution volume of $50 \mu \mathrm{L}$

We performed rRT-PCR using PowerCheck 2019-nCoV for all pooled specimens. The interpretation guideline by the manufacturer for SARS-CoV-2 positivity was a $C_{t}$ cutoff of $\leq 35$ for a single specimen; however, we assessed every amplified curve throughout 40 total PCR cycles. For either the $E$ or $R d R p$ gene, when we observed any amplified curve before the end of the 40 amplification cycles, we interpreted the result as positive for the pooled specimens. When we observed no amplification curves for both genes, we interpreted the result as negative.

We performed all statistical analyses with MedCalc version 19.2.1 (MedCalc Software Ltd, https:// www.medcalc.org). The distribution of $C_{t}$ values in individual specimens (Figure 1) showed negative skewness. In total, $61 \%$ of confirmed cases had $C_{t} \geq 30$, which was near the cutoff value. We selected positive samples for pooling according to this distribution pattern.

The pooled positive specimens had $100 \%$ sensitivity in pool sizes 2, 4, and 6 and $97 \%-99 \%$ sensitivity in pool sizes 8,10 , and 16 (Table). To ensure a conservative estimation of sensitivity, we calculated the cumulative sensitivities on the assumption that the false-negative results that occurred in smaller pool sizes could also occur in larger pool sizes.

\begin{tabular}{lcccc}
\hline \multicolumn{4}{l}{ Table. Test performance of pooled specimens compared with individual specimens for severe acute respiratory syndrome coronavirus 2 } \\
\hline No. specimens in pool & $\begin{array}{c}\text { Amplification in } E \text { or } \\
\text { RdRp gene, } \%\end{array}$ & No amplifications & $\begin{array}{c}\text { Sensitivity of pools, \% } \\
(95 \% \text { Cl) }\end{array}$ & $\begin{array}{c}\text { Cumulative sensitivity, } \\
\%{ }^{*}\end{array}$ \\
\hline 2 & 100 & 0 & $100(96-100)$ & 100 \\
4 & 100 & 0 & $100(96-100)$ & 100 \\
6 & 100 & 0 & $100(96-100)$ & 100 \\
8 & 97 & 3 & $97(92-99)$ & 97 \\
10 & 99 & 1 & $99(95-100)$ & 96 \\
16 & 96 & 4 & $96(90-98)$ & 92 \\
\hline
\end{tabular}

${ }^{*}$ Calculated sensitivity based on the accumulated discrepancy numbers under the dilution fold 
Therefore, every negative result that occurred in smaller pool sizes was included in the calculation of cumulative sensitivities in larger pool sizes. The cumulative sensitivities of pool size 6 was $100 \%$, of $8,97 \%$, of $10,96 \%$, and of $16,92 \%$. The clinical specificity of pool size 16 was $97 \%$ (58/60, 95\% CI $87 \%$ $99 \%$ ). The mean $C_{t}$ values increased for both the $E$ and $R d R p$ genes as the pool size increased (Figure 2; Appendix Figure).

\section{Conclusions}

We evaluated the clinical sensitivity and specificity of SARS-CoV-2 rRT-PCR using pooled upper respiratory specimens from confirmed cases. Because pooled specimens are expected to be used as a screening tool, the clinical sensitivity of pooled specimens at a given pool size is especially important.

A limitation of previous studies is that the $C_{t}$ values of positive specimens from patients at the time of diagnosis were not considered in the study design. The $C_{t}$ values of specimens in previous studies were relatively low (6). Because specimens with high $C_{t}$ values, meaning low virus titers, are expected to be vulnerable to pooling, the distribution of $C_{t}$ values in the actual population should be reflected when determining the pool size. We analyzed the actual distribution of $C_{t}$ values from 4,364 initially confirmed cases, and the distribution showed skewness with regard to the PCR cutoff value.

Yelin et al. (4) suggested that the pool size using RNA extracts could be $\leq 64$; however, we do not recommend increasing the pool size to 64 , corresponding to a theoretical increase in $C_{t}$ values of 6 , given the associated loss in sensitivity; doing so may cause false negative results.

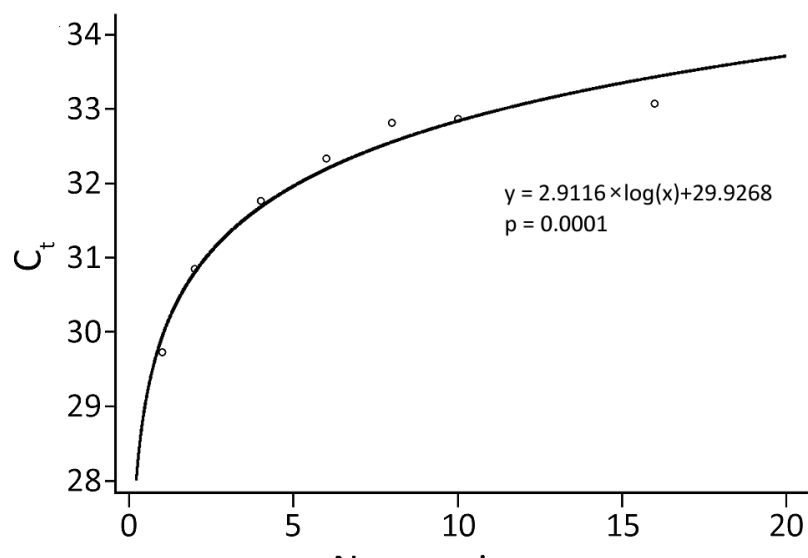

No. specimens

Figure 2. Mean $C_{t}$ values of $R d R p$ genes of 50 specimens from coronavirus disease patients in South Korea by pool size. The trend line shows logarithmic regression. $\mathrm{C}_{\mathrm{t}}$, cycle threshold.
The pooling strategy showed efficiency when the positive rates in the population were low (7). We showed clinical sensitivities and cumulative sensitivities of the pooled specimens that were sampled after stratification by data, including low viral titers. On the basis of our results, we recommend pooling $\leq 6$ specimens in clinical practice. Pooling $>6$ specimens might cause false-negative results, considering the observed abundance of specimens with high $C_{t}$ values in the population.

This study has some limitations. First, the analytical performance of the PCR kit used has not been evaluated fully because it is one of the earliest available commercial PCR kits that received the Emergency Use Authorization in Korea. Second, the positive cutoff in the kit was a $C_{t}$ value $\leq 35$ within 40 amplification cycles. Therefore, this study did not include individual specimens with a $C_{t}$ value $>35$, which is interpreted as an inconclusive result by this kit. Third, we did not evaluate cost-effectiveness on the basis of the hypothesized prevalence. Last, we did not evaluate the effect of specimen volume in the pools; increasing the input volume from each specimen may improve the sensitivity of the pooling test.

Our protocol will be helpful for screening persons in groups at high risk for COVID-19 infection quickly and quarantining those confirmed positive, even in situations with limited time and test resources. Epidemiologic factors should be considered when choosing an adequate pooling number. Symptomatic case-patients should be tested individually without pooling to enable effective and timely action. We have included practical guidelines for specimen-pooling procedures in the Appendix.

\section{Acknowledgments}

We thank Mallikarjun Handigund for his contribution in generating the data for this study, and Joongyub Lee for his contribution to the statistical analysis. We thank all laboratory technologists in the Department of Molecular Diagnostics at National Medical Center, Jeonbuk National University Hospital, and Seoul Medical Center for their contributions in generating the data for this study.

Author contributions: S.W.L. and K.H.H. take responsibility for the integrity of the data and the accuracy of the data analysis. S.Y.K. and J.L. contributed equally to this study.

\section{About the Author}

Dr. Kim is a medical doctor and director of the Department of Laboratory Medicine, National Medical Center, Seoul, South Korea. Her research interests include 
molecular diagnostics and infectious diseases. Dr. Lee is a medical doctor and assistant professor in the Department of Laboratory Medicine, Jeonbuk National University Medical School and Hospital, Jeonju, South Korea. His research interests include clinical microbiology and infectious disease.

\section{References}

1. Zhu N, Zhang D, Wang W, Li X, Yang B, Song J, et al.; China Novel Coronavirus Investigating and Research Team. A novel coronavirus from patients with pneumonia in China, 2019. N Engl J Med. 2020;382:727-33. https://doi.org/ 10.1056/NEJMoa2001017

2. World Health Organization. WHO Director-General's opening remarks at the media briefing on COVID-19-11 March 2020. Geneva: The Organization; 2020 [cited 2020 Aug 19]. https://www.who.int/dg/speeches/detail/ who-director-general-s-opening-remarks-at-the-media-briefing-on-covid-19---11-march-2020

3. Hong KH, Lee SW, Kim TS, Huh HJ, Lee J, Kim SY, et al. Guidelines for laboratory diagnosis of coronavirus disease 2019 (COVID-19) in Korea. Ann Lab Med. 2020;40:351-60. https:/ / doi.org/10.3343/alm.2020.40.5.351
4. Yelin I, Aharony N, Shaer Tamar E, Argoetti A, Messer E, Berenbaum D, et al. Evaluation of COVID-19 RT-qPCR test in multi-sample pools. Clin Infect Dis. 2020 May 2 [Epub ahead of print]. https://doi.org/10.1093/cid/ ciaa531

5. Hogan CA, Sahoo MK, Pinsky BA. Sample pooling as a strategy to detect community transmission of SARS-CoV-2. JAMA. 2020;323:1967-9. https://doi.org/ 10.1001/jama.2020.5445

6. Lohse S, Pfuhl T, Berkó-Göttel B, Rissland J, Geißler T, Gärtner B, et al. Pooling of samples for testing for SARS-CoV-2 in asymptomatic people. Lancet Infect Dis. 2020 Apr 28 [Epub ahead of print]. https://doi.org/10.1016/ S1473-3099(20)30362-5

7. European Centre for Disease Prevention and Control. Methodology for estimating point prevalence of SARSCoV-2 infection by pooled RT-PCR testing. Stockholm: The Centre; 2020 [cited 2020 Aug 19]. https://www.ecdc.europa.eu/en/publications-data/ methodology-estimating-point-prevalence-sars-cov-2infection-pooled-rt-pcr

Address for correspondence: Ki Ho Hong, Department of Laboratory Medicine, Seoul Medical Center, 156 Sinnae-ro, Jungnang-gu, Seoul, 02053, South Korea; email: kihohongmd@gmail.com

\section{EID Podcast Meningitis in U.S. Colleges}

The number of reported outbreaks of meningococcal disease at U.S. universities has increased in recent years, despite the availability of vaccines. So why are college students still at increased risk for this potentially deadly disease?

\section{In this EID podcast, Dr. Heidi Soeters, a CDC epidemiologist, discusses the prevalence of meningitis at U.S. universities.}

\section{Visit our website to listen: EMERCING} https://tools.cdc.gov/medialibrary/index.aspx\#/media/id/397588 INFECTIOUS DISEASES 